Major Article

\title{
Intervention to reduce the incidence of surgical site infection in spine surgery
}

\author{
Laia Castellà BN ${ }^{\mathrm{a}, \mathrm{b}}$, Nieves Sopena MD ${ }^{\mathrm{b}, \mathrm{c}, \mathrm{d}, *}$, David Rodriguez-Montserrat $\mathrm{MB}^{\mathrm{e}}$, \\ ${ }^{a}$ Germans Trias i Pujol University Hospital, Badalona, Spain \\ ${ }^{\mathrm{b}}$ IGTP Health Sciences Research Institute, Germans Trias i Pujol University Hospital, Badalona, Spain \\ ' Infectious Diseases Department, Germans Trias i Pujol University Hospital, Badalona, Spain \\ ${ }^{\mathrm{d}}$ Faculty of Medicine, Universitat Autònoma de Barcelona, Cerdanyola del Vallès, Barcelona, Spain \\ e Orthopedic Surgery Department, Germans Trias i Pujol University Hospital, Badalona, Spain \\ ${ }_{\mathrm{f}}$ Bellvitge Biomedical Research Institute, L'Hospitalet de Llobregat, Barcelona, Spain \\ ${ }^{g}$ Fundamental Care and Medical-Surgical Nursing Department, School of Nursing, University of Barcelona, Barcelona, Spain \\ ${ }^{\mathrm{h}}$ Microbiology Department, North Metropolitan Clinical Laboratory, Germans Trias i Pujol University Hospital, Badalona, Spain \\ i Preventive Medicine Department, Germans Trias i Pujol University Hospital, Badalona, Spain
} Sergio Alonso-Fernández BN ${ }^{\mathrm{a}, \mathrm{b}, \mathrm{f}, \mathrm{g}}$, Jose María Cavanilles $\mathrm{MB}^{\mathrm{e}}$, Miquel Iborra $\mathrm{MB}^{\mathrm{e}}$, Ana Ciercoles BN ${ }^{\mathrm{a}}$, Ana Pulido BN ${ }^{\mathrm{a}}$, Montserrat Gimenez MD ${ }^{\mathrm{d}, \mathrm{h}}$, Jose Antonio Hernandez Hermoso MD ${ }^{\mathrm{d}, \mathrm{e}}$, Irma Casas MD ${ }^{\mathrm{d}, \mathrm{i}}$

Key Words:

Infection control

Surgical site infection

Intervention

\begin{abstract}
Background: This study examines the incidence, characteristics, and risk factors of surgical site infections (SSIs) after spine surgery and evaluates the efficacy of a preventive intervention.

Methods: This was a quasi-experimental pretest/posttest study in patients undergoing spinal surgery in an orthopedic surgery department from December 2014 to November 2016. Based on the results of the study, we revised the preventive protocol with modification of wound dressing, staff training, and feedback. SSI rates were compared between the pre-intervention (December 2014 to November 2015) and post-intervention (December 2015 to November 2016) periods. The risk factors were analyzed using univariate and multivariate analyses. Results: Of the 139 patients included, 14 cases of SSI were diagnosed, with a significant decrease in the incidence of SSIs from the pre-intervention period to the post-intervention period ( $19.4 \%$ vs $2.6 \% ; P=.001)$. The etiology was known in 13 cases, with enteric flora being predominant in the pre-intervention group. Univariate analysis showed that age, body mass index, days until sitting and ambulation, and incontinence were statistically significant risk factors. After multivariate analysis, only body mass index and days until ambulation remained significant. When the effect of intervention was adjusted with other risk factors, this variable remained statistically significant.

Conclusions: An intervention that includes modification of wound dressing and early mobilization, as well as staff awareness training, monitoring, and feedback, allowed a significant reduction in the incidence of SSI following spinal surgery, particularly infections caused by enteric flora.
\end{abstract}

(c) 2019 Association for Professionals in Infection Control and Epidemiology, Inc. Published by Elsevier Inc. All rights reserved.

\section{INTRODUCTION}

Surgical site infections (SSIs) in spinal surgery are a potentially serious complication that increases morbidity, mortality, hospital

\footnotetext{
*Address correspondence to Nieves Sopena, MD, Infectious Diseases Department, Germans Trias i Pujol University Hospital, Carretera de Canyet s/n, 08019 Badalona, Spain. E-mail address: nsopena.germanstrias@gencat.cat (N. Sopena).

Conflicts of interest: None to report.

Funding/support: Supported by the "Trias Talents" aid program of the Germans Trias i Pujol Research Institute and University Hospital.
}

stays of patients, and health costs. Reports in the literature indicate that the incidence of spinal SSI varies between $0.5 \%$ and $18.8 \%$, with the main risk factors for infection depending on the patient (obesity, diabetes, smoking, corticosteroid therapy, previous hospital stay), the surgical procedure (instrumentation, implants, number of levels operated, site of surgery, duration of the intervention, dural tear, revision surgery, need for transfusion), and the postoperative period (days of drainage, incontinence, prolonged bed rest). ${ }^{1-12}$

SSIs after spine surgery usually occur due to direct inoculation during the surgical procedure and less frequently by hematogenous spread or early postoperative contamination. Staphylococcus aureus is 
the most frequent etiology, causing $12 \%-65 \%$ of infections, followed by coagulase-negative staphylococci, especially in the presence of prosthetic implants. In contrast, Gram-negative organisms tend to cause infections in low lumbar interventions due to the proximity of the perineal area, as well as in incontinent patients or patients with prolonged hospitalization prior to the intervention. ${ }^{3,6}$

Some studies show that up to $60 \%$ of SSIs could be prevented by applying strategies that include a set of evidence-based measures. ${ }^{13}$ Education in preventive measures and providing continuous feedback on SSI rates to surgical and perioperative personnel are an essential part of such a preventive strategy. In December 2014, an increase in SSI cases following spinal surgery was detected in the traumatology service of our hospital, when the incidence rate rose to $7.2 \%$. In response to this increase, the present study was undertaken to determine the incidence, characteristics, and risk factors of SSIs in spinal surgery and to evaluate the effectiveness of a preventive intervention.

\section{METHODS}

A quasi-experimental, pretest/posttest study with a historical control group was performed in patients undergoing spinal surgery at the trauma service of the Germans Trias i Pujol University Hospital in Badalona, Spain.

\section{Study population and setting}

We included all 139 patients who had undergone lumbar spinal surgery in the trauma service of Germans Trias i Pujol University Hospital from December of 2014 to November of 2016. Germans Trias i Pujol is a 550-bed tertiary teaching hospital located in Badalona, Spain, which serves as a reference center for the more than 800,000 citizens of the Barcelonès Nord and Maresme catchment areas. The trauma service's team of 3 surgeons performs about 70 spinal operations annually. Reasons for spinal surgery include disc herniation, spinal stenosis, spondylolisthesis, scoliosis, kyphosis, and trauma. The hospital has a multidisciplinary infection control team.

Two periods of study were defined: the pre-intervention period from December 2014 to November 2015 and the post-intervention period from December 2015 to November 2016. The 62 patients who had undergone surgery in the pre-intervention period constituted the historical control group. The remaining 77 patients who underwent surgery after November 2015 constituted the experimental group in that they prospectively were assigned to receive the intervention described below. This clinical research was approved by the Clinical Research Ethics Committee for Research Projects of the Germans Trias i Pujol University Hospital (PI-16-154).

\section{Intervention}

The intervention consisted of reviewing and updating the preventive protocol of SSIs in spinal surgery with participation of the professionals involved. The main preventive measures are listed in Table 1 . The dressing of the surgical wound was modified with the application of an OPSITE Post-Op Visible occlusive dressing (Smith +Nephew; London, UK). ${ }^{14}$ This is an adhesive dressing consisting of absorbent hydrocellular foam in the form of a grating with a waterproof, transparent film that allows visualization of the wound without the need to lift the dressing. The dressing was maintained for 5-7 days depending on its degree of saturation and the absence of complications, as monitored by infection control nurses. The hygiene of the bedridden patient was carried out following the established protocol before the occlusive dressing was removed to allow curing.
Table 1

Preventive measures

\begin{tabular}{lll}
\hline Time & Measures & Description \\
\hline Preoperative & Preoperative optimization & Diabetes, obesity, smoking \\
& Antiseptic shower & Chlorhexidine soap \\
& Chlorhexidine with alcohol \\
& Hair removal if necessary & Hair removal with clippers \\
Intraoperative & Checklist & WHO checklist \\
& Hygiene standards and & Hospital protocol \\
& uniformity & \\
& Antibiotic prophylaxis & Hospital protocol \\
& Normothermia & Temperature control \\
& Glycemic control & and warming \\
Postoperative & Wound dressing & OPSitE Visible dressing \\
& Hygiene of the bedridden & Hospital protocol \\
& patient & Sitting and ambulation \\
& Early mobilization protocol & within 24-48 h \\
\hline
\end{tabular}

WHO, World Health Organization.

\section{Data collection}

The main outcome variable was surgical infection. Surgical infection was defined according to Centers for Disease Control and Prevention criteria, ${ }^{13}$ whereby an infection was considered to be a SSI when it occurred at the site of the surgery within 30 days after the operation or within 1 year in the presence of an implant. A deep surgical site infection was defined as an infection involving the deep soft-tissue muscle and fascia, in contrast to a superficial infection with only skin and subcutaneous tissue affected.

The independent variables included sociodemographic characteristics (age, sex), intrinsic risk factors (smoking habit, body mass index [BMI], diabetes, American Society of Anesthesiologists [ASA] classification, anemia, previous spinal surgery), and perioperative and postoperative extrinsic factors (preoperative stay, elective vs urgent surgery, instrumentation, bone graft, adequate antibiotic prophylaxis, transfusion, duration of surgery, number of levels, drainage use and duration, days to sitting and ambulation, urinary and fecal incontinence), as well as intervention with a package of measures including application of the new wound dressing. The data were collected retrospectively in the pre-intervention period and prospectively during the post-intervention period, in both cases from the patient's computerized clinical history in the SAP (Walldorf, Germany) health care environment, as well as the nursing care registry of the Gacela Care program.

\section{Statistical analysis}

Quantitative variables were described in terms of mean and standard deviation; for variables that did not follow a normal law, median and minimum and maximum values were used. Categorical variables were expressed in absolute frequencies and percentages. A univariate analysis of the relationship between the variables and surgical infection was performed using the $\chi^{2}$ test, Fisher's exact test, and $t$-test or the Mann-Whitney $U$ test. Subsequently, a multivariate analysis was carried out using binary logistic regression, entering as the main independent variable the intervention period and as possible confounding variables those detected in the univariate analysis. The Hosmer-Lemeshow test was performed to analyze the goodness of fit of the final model. SPSS Statistics 25 (IBM; Armonk, NY) was used, and a $P<.05$ bilateral was used to detect statistically significant differences.

\section{RESULTS}

As noted above, of the 139 patients who underwent spinal surgery during the study period, 62 were in the pre-intervention period (the 
control group) and 77 in the post-intervention period. The sociodemographic characteristics and risk factors of patients in the pre-intervention and post-intervention groups are shown in Table 2. Of the total, 70 were men, with an average age of $55.3 \pm 16.5$ years; $34.5 \%$ were smokers with a mean BMI of $26.5 \pm 5.1$ and a mean hemoglobin level of $13.6 \pm 1.6 \mathrm{~g} / \mathrm{dL}$. Only $18 \%$ presented an ASA classification of III or IV. Surgery was elective in most cases (97.1\%). The median length of time between admission and intervention was 1 day (range, $0-41$ ), and $46.8 \%$ of the patients were admitted on the day of the surgery. The location of surgery was lumbosacral in 109 cases $(78.4 \%)$, thoracolumbar in 20 (14.4\%), dorsolumbosacral in 5 (3.6\%), thoracic in 8 (5.8\%), and cervical in 4 (2.9\%); $69.1 \%$ underwent an instrumented procedure, and in $68.3 \%$ a bone graft was used.

No statistically significant differences were found between the patients of the 2 groups in most of the demographic characteristics and risk factors. The variables that were significant were the type of surgery (elective vs urgent) and instrumentation, with a higher percentage in both cases being found among the patients in the postintervention group (Table 2).

Patients with instrumentation were older than those without instrumentation $(57.7 \pm 17.4$ vs $49.9 \pm 13$ years; $P=.01)$. They also had a higher frequency of some risk factors such as ASA $\geq 3(23.9 \%$ vs $9.5 \% ; P=.05)$, number of levels $>2(39.8 \%$ vs $7,1 \% ; P<.001)$, duration of surgery (222.97 \pm 78.64 vs $113.27 \pm 69.71 \mathrm{~min} ; P<.001)$, transfusions $(17.4 \%$ vs $4.8 \% ; P<.001)$, and postoperative drainage $(98.9 \%$ vs $72.1 \% ; P=.04)$. Moreover, they had more days of drainage $(1.9 \pm 0.5 \mathrm{vs}$ $1.4 \pm 0.7$ days; $P<.001)$ and a greater delay to ambulation $(4.1 \pm 4.1$ vs $1.6 \pm 0.7$ days; $P=.005)$.

Adherence to preventive measures was only evaluated for some measures, such as adequate antibiotic prophylaxis and early mobilization. In the post-intervention group, wound dressing with OPSITE Visible was performed in 71 of the patients in the postoperative period (92.2\%). Adherence to bath and preparation of the skin protocols occurred in most cases in both periods. Compliance with other specific preventive measures such as normothermia and glycemic control has not been studied.

During the study period, 14 patients presented SSIs, of which 6 were superficial and 2 organ space. The incidence of SSI was 19.4\% in the preintervention group (12 cases) and $2.6 \%$ in the post-intervention group
(2 cases), representing a statistically significant decrease $(P=.001)$. All of the infections occurred in spinal surgery including lumbar level with posterior approach.

The microbiological diagnosis was obtained in 13 cases (92.8\%): $S$ aureus (4 cases), Escherichia coli (3 cases), Pseudomonas aeruginosa ( 2 cases), Morganella morganii (1 case), and 3 cases of polymicrobial infections: M. morganii + Klebsiella pneumoniae + Enterococcus faecalis (1 case), Enterobacter spp $+E$ faecalis (1 case), and $E$ faecalis $+S$ coagulase negative (1 case). The infections in the pre-intervention control group were caused by Gram-negative bacilli in 8 cases (66.6\%), and in 3 cases (25\%) they were polymicrobial. The 2 infections in the post-intervention group were caused by $S$ aureus. Eight cases (57.1\% of the SSIs) were diagnosed during hospitalization, and the other cases were readmitted due to infection. The mean time from surgery to the diagnosis of infection was $14.2 \pm 9.25$ days (range, 2-32); the mean time was $12.9 \pm 9.1$ days (range, 2-32) in the pre-intervention group and $23 \pm 4.2$ days (range, 20-26) in the post-intervention group. Eleven of the 14 patients (78.5\%) who presented an SSI required 1 ( 9 cases) or 2 ( 2 cases) reoperations to treat the infection. One case required removal of the instrumentation material. No patient died from the infection. Patients with SSIs presented a total mean stay greater than patients without infection (median, 8 days; range, 4-271 vs median, 6 days; range, 2-62; $P=.008)$.

The risk factors of patients with SSIs that were significant in the univariate analysis were mean age ( $63.9 \pm 10.7$ vs $54.3 \pm 16.0$ years; $P=.007)$, mean BMI $(29.6 \pm 5.6$ vs $26.2 \pm 4.5 ; P=.01)$, median of days until the start of sitting (2 days; range, 1-32 vs 3 days; range, $1-8 ; P=.006)$, and the median of days until walking (4 days; range, $2-30$ vs 2 days; range, $1-9 ; P=.001)$. Urinary and fecal incontinence in the postoperative period ( $14.3 \%$ vs $1.6 \% ; P=.050$ ) were nearly significant, as were the variables significantly associated with the development of SSI. The mean duration of surgery was longer in patients with SSIs $(228.9 \pm 88.6$ vs $182.8 \pm 90.1 \mathrm{~min} ; P=.07)$, although it was not statistically significant. Patients with SSIs presented a higher frequency of diabetes ( $35.7 \%$ vs $18.4 \%$ ) and perioperative transfusions (28.6\% vs $11.7 \%$ ), as well as a higher number of operated levels (>2 levels in $15 \%$ vs $8.4 \%$ ), although the differences were not statistically significant.

Table 2

Comparison of sociodemographic and clinical characteristics of patients in pre-intervention and post-intervention groups

\begin{tabular}{|c|c|c|c|c|}
\hline Variable & Total $(\mathrm{N}=139)$ & Pre-intervention group $(\mathrm{N}=62$ ) & Post- intervention group $(\mathrm{N}=77$ ) & $P$ value \\
\hline Age $(y)$, mean $\pm S D$ & $55.3 \pm 16.5$ & $55.3 \pm 16.6$ & $55.2 \pm 16.5$ & .958 \\
\hline Gender (male), n (\%) & $70(50.4)$ & $34(54.8)$ & $36(46.8)$ & .343 \\
\hline Current smoking, n (\%) & $48(34.5)$ & $24(38.7)$ & $24(31.2)$ & .353 \\
\hline Body mass index, mean $\pm S D$ & $26.5 \pm 5.1$ & $26.1 \pm 5.1$ & $26.8 \pm 5.1$ & .391 \\
\hline Diabetes, $\mathrm{n}(\%)$ & $28(20.1)$ & $11(17.7)$ & $17(22.1)$ & .526 \\
\hline ASA classification $\geq 3, \mathrm{n}(\%)$ & $25(18)$ & $9(15.3)$ & $16(22.5)$ & .294 \\
\hline Preoperative hemoglobin, mean \pm SD & $13.6 \pm 1.6$ & $13.6 \pm 1.6$ & $13.5 \pm 1.6$ & .610 \\
\hline Preoperative stay (d), median (minimum-maximum) & $1(0-41)$ & $1(0-17)$ & $1(0-41)$ & .528 \\
\hline Admission the same day of surgery, $\mathrm{n}(\%)$ & $65(46.8)$ & $27(43.5)$ & $38(49.4)$ & .496 \\
\hline Elective vs urgent surgery, $\mathrm{n}(\%)$ & $135(97.1)$ & $58(93.5)$ & $77(100)$ & .024 \\
\hline Surgical site lumbar, n (\%) & $127(91.4)$ & $59(95.2)$ & $68(88.3)$ & .153 \\
\hline Number of levels > 2, n (\%) & $40(29.6)$ & $18(29)$ & $22(30.1)$ & .889 \\
\hline Instrumentation, $\mathrm{n}(\%)$ & $96(69.1)$ & $37(59.7)$ & $59(76.7)$ & .032 \\
\hline Bone grafts, $\mathrm{n}(\%)$ & $95(68.3)$ & $38(61.3)$ & $57(74)$ & .109 \\
\hline Adequate antibiotic prophylaxis, mean \pm SD & $134 \pm 96.4$ & $61 \pm 98.4$ & $73 \pm 98.4$ & .105 \\
\hline Transfusion, n (\%) & $18(12.9)$ & $9(15.5)$ & $9(11.8)$ & .536 \\
\hline Duration of surgery (min), mean $\pm S D$ & $187.4 \pm 90.7$ & $182.2 \pm 97.8$ & $191.7 \pm 84.9$ & .540 \\
\hline Drainage use, n (\%) & $125(89.9)$ & $53(86.9)$ & $72(93.5)$ & .186 \\
\hline Days of drainage use, median (minimum-maxim) & $2(1-4)$ & $2(1-3)$ & $2(1-4)$ & .907 \\
\hline Days to sitting, median (minimum-maxim) & $2(1-32)$ & $2(1-8)$ & $2(1-5)$ & .749 \\
\hline Days to ambulation, median (minimum-maxim) & $3(1-30)$ & $2(1-30)$ & $2(1-6)$ & .830 \\
\hline Urine incontinence, $\mathrm{n}(\%)$ & $4(2.5)$ & $2(3.2)$ & $2(2.6)$ & 610 \\
\hline Fecal incontinence, $\mathrm{n}(\%)$ & $4(2.5)$ & $2(3.2)$ & $2(2.6)$ & 610 \\
\hline
\end{tabular}

ASA, American Society of Anesthesiologists; $S D$, standard deviation. 
The statistically significant variables in the univariate analysis were entered into the multivariate logistic regression model, except urinary and fecal incontinence, as they only occurred in 4 cases. The mean time of surgical duration was also entered. The variables that were statistically significant with the presence of SSI were BMI and days until ambulation (Table 3).

Subsequently, another multivariate logistic regression model was performed to analyze the impact of the intervention on SSI, adjusted for BMI and days until ambulation. After adjusting for these confounding factors, the pre-intervention group showed an adjusted odds ratio of 7.71 (95\% confidence interval, 1.44-41.1). The results of the Hosmer-Lemeshow test showed that the final model was a good fit to the data $\left(\chi^{2}=7.19 ; P=.56\right)($ Table 4$)$.

\section{DISCUSSION}

The implementation of a multidisciplinary intervention that included revision of the preventive protocol with the modification of wound dressing, staff training, and use of surveillance feedback from results was associated with a $78.1 \%$ decrease in the incidence of surgical infection in spinal surgery in the trauma service. The majority of surgical procedures were elective and $69.1 \%$ instrumented. The patients were comparable in the pre-intervention and post-intervention groups in terms of risk factors, although the percentage of procedures with instrumentation was significantly higher in the post-intervention group and that of elective surgery in the pre-intervention control group. The incidence of SSI decreased from $19.4 \%$ in the pre-intervention group to $2.6 \%$ in the post-intervention group, which is comparable to the overall infection rate in some previous studies that included instrumentation. ${ }^{2,3}$

The infections that occurred in the pre-intervention period were mainly caused by microorganisms that are part of the enteric flora such as Gram-negative bacilli. This fact suggested perioperative contamination of the wound as the source of these pathogens, considering that most of the interventions were located in the lumbar area. ${ }^{3}$ In contrast, the infections that occurred in the post-intervention period were caused by skin commensal flora such as $S$ aureus, which is the predominant etiology in most studies. ${ }^{6,9}$

Coinciding with other studies, SSI increased the length of stay (by about 2 days), as well as the rate of reoperation, which was necessary for most of the infected patients, and therefore the associated health costs. ${ }^{6,11}$ The risk factors associated with SSI following spine surgery in our study were obesity and days to ambulation. Other factors such as age, days to sitting, and urinary and fecal incontinence that were significant in the univariate analysis did not maintain their significance in the multivariate analysis.

It is noteworthy that half of the patients in our study were overweight, and a quarter were obese. Obesity is one of the most prevalent health problems in the developed world. ${ }^{15}$ Many studies ${ }^{2,5,15-20}$ have shown that being overweight increases the risk of SSI in spinal surgery, possibly due to its association with other comorbidities, the increase in operative time, greater bleeding, a higher probability of underdosing of antibiotic prophylaxis, and delayed mobilization in the postoperative period. Consequently, weight loss should be included in the list of optimization measures prior to spinal surgery. ${ }^{21}$

Early mobilization is a fundamental component of multimodal strategies for fast postoperative recovery. In a study carried out by Adogwa et $\mathrm{al}^{22}$ in patients older than 65 years undergoing spinal surgery, early postoperative mobilization reduced the incidence of postoperative complications and length of stay and contributed to improved functionality at discharge. Although this study did not demonstrate a link between time to mobilization and the incidence of SSI, the delay in mobilization in spinal surgery could increase the risk of SSI by increasing the pressure in the surgical area and favoring contamination in the dorsal and lumbar localization procedure. The early mobilization coupled with adequate pain control will likely help to reduce SSIs in spinal surgery.

Older people often have other comorbidities and malnutrition, as well as increased susceptibility to infection, ${ }^{23}$ and several studies have shown age to be a risk factor for SSI following spine surgery. Nevertheless, age was not significant in the multivariate study carried out here.

Likewise, in contrast to a previous study, our results did not show urinary incontinence to be an independent risk factor for SSI following spinal surgery, probably due to the small number of patients with this risk factor and interaction with other factors. ${ }^{4}$ Urinary incontinence has been associated with asymptomatic bacteriuria and, along with fecal incontinence, favors contamination of the surgical wound by Gram-negative bacteria in part by hindering adhesion of the dressing. ${ }^{3}$

Compared with previous studies, ${ }^{1-12}$ other factors such as the number of levels, duration of the intervention, diabetes, transfusion, or use of drainage or instrumentation were not significant in our study, possibly due to confusion with other variables. However, $20 \%$ of the patients in the present study and almost $36 \%$ of those who presented a SSI were diabetic. In this regard, it should be noted that preoperative optimization of diabetes and perioperative control of blood glucose are prominent elements in guidelines for the prevention of

Table 3

Multivariate analysis of risk factors associated with surgical site infections after spine surgery

\begin{tabular}{|c|c|c|c|c|}
\hline Variable & $\operatorname{Exp}(\beta)$ & 95\% CI (lower limit) & 95\% CI (upper limit) & $P$ value \\
\hline Age & 1.02 & 0.97 & 1.08 & .374 \\
\hline Body mass index $\left(\mathrm{kg} / \mathrm{m}^{2}\right)$ & 1.17 & 1.03 & 1.32 & .015 \\
\hline Surgery duration (min) & 1.0 & 0.99 & 1.01 & .575 \\
\hline Days to sitting & 1.03 & 0.80 & 1.31 & .806 \\
\hline Days to ambulation & 1.26 & 0.99 & 1.60 & .059 \\
\hline
\end{tabular}

CI, confidence interval.

Table 4

Impact of intervention on surgical site infections adjusted for body mass index and days to ambulation

\begin{tabular}{|c|c|c|c|c|}
\hline Variable & $\operatorname{Exp}(\beta)$ & 95\% CI (lower limit) & 95\% CI (upper limit) & $P$ value \\
\hline Pre-intervention period & 7.71 & 1.44 & 41.1 & .017 \\
\hline Body mass index & 1.18 & 1.04 & 1.34 & .007 \\
\hline Days to ambulation & 1.34 & 1.03 & 1.74 & .026 \\
\hline
\end{tabular}

CI, confidence interval. 
SSIs. ${ }^{23}$ The rates of infection are higher in instrumented procedures. ${ }^{1}$ However, instrumentation was not a risk factor for infection in our study, possibly because it occurred in patients with other risk factors for infection such as age, duration of surgery, delay in sitting, and transfusion.

The impact of the intervention on SSIs remained statistically significant when it was adjusted for BMI and days until ambulation. The intervention included several features that increased compliance with the main SSI preventive measures. First, the health professionals involved in both surgery and recovery were given information that increased their awareness of the importance of preventing this infection. $^{24,25}$ Second, feedback from postoperative surveillance of patients was provided to surgeons, a methodology that has been shown to be fundamental in the prevention of SSIs. ${ }^{12}$ Third, the use of a transparent and impermeable occlusive dressing allowed the wound to be monitored without the need to change the dressing. This measure, which limits potential wound contamination, helped to reduce the rate of infection by enteric flora. ${ }^{14}$

One limitation of this study is its quasi-experimental design, as it cannot be guaranteed that the results obtained are due to the intervention itself rather than to a specific measure, other interventions, or uncontrolled factors. Likewise, the small size of the sample of infected patients means that the statistical power of the study is low. Compliance with specific preventive measures has not been studied, so we cannot know the particular impact of each of them on the reduction of SSIs. Moreover, further studies would be useful to determine the most suitable dressing for this type of surgery.

\section{CONCLUSIONS}

An intervention that includes a revision of the protocol for treating spinal surgery patients, modification of wound healing procedures, and early mobilization, as well as staff training, monitoring of recovering patients, and providing feedback from surveillance results, can allow a significant reduction in the incidence of SSIs following spinal surgery, especially infections caused by enteric flora. This suggests that adoption of the sort of surgical wound dressing employed here should be included in the protocols intended to prevent SSIs.

\section{Acknowledgments}

We acknowledge the contributions of Ms. Antonia Salmeron, as well as all the nurses and doctors of the Germans Trias i Pujol University Hospital trauma service and operation room.

\section{References}

1. Parchi PD, Evangelisti G, Andreani L, Girardi F, Darren L, Sama A, et al. Postoperative spine infections. Orthop Rev (Pavia) 2015;7:5900.
2. Gu W, Tu L, Liang Z, Wang Z, Aikenmu K, Chu G, et al. Incidence and risk factors for infection in spine surgery: a prospective multicenter study of 1764 instrumented spinal procedures. Am J Infect Control 2018;46:8-13.

3. Chahoud J, Kanafani C, Kanj SS. Surgical site infections following spine surgery: eliminating the controversies in the diagnosis. Front Med 2014;1:7.

4. Radcliff KE, Neusner AD, Millhouse PW, Harrop JD, Kepler CK, Rasouli MR, et al. What is new in the diagnosis and prevention of spine surgical site infections. Spine J 2015;15:336-47.

5. Olsen MA, Mayfield J, Lauryssen C, Polish LB, Jones M, Vest J, et al. Risk factors for surgical site infection in spinal surgery. J Neurosurg 2013;98:149-55.

6. Cassir N, De La Rosa S, Melot A, Touta A, Troude L, Loundou A, et al. Risk factors for surgical site infections after neurosurgery: a focus on the postoperative period. Am J Infect Control 2015;43:1288-91.

7. Meng F, Cao J, Meng X. Risk factors for surgical site infections following spinal surgery. J Clin Neurosci 2015;22:1862-6.

8. Rao SB, Vasquez G, Harrop J, Maltenfort M, Stein N, Kaliyadan G, et al. Risk factors for surgical site infections following spinal fusion procedures: a case-control study. Clin Infect Dis 2011:53:686-92.

9. Schimmel JJP, Horsting PP, de Kleuver M, Wonders G, van Limbeek J. Risk factors for deep surgical site infections after spinal fusion. Eur Spine J 2010;19:1711-9.

10. Pesenti S, Pannu T, Andres-Bergos J, Lafage R, Smith JS, Glassman S, et al. What are the risk factors for surgical site infection after spinal fusion? A meta-analysis. Eur Spine J 2018;27:2469-80.

11. Apisarnthnarak A, Jones M, Waterman BM, Caroll CM, Bernardi R, Fraser VJ. Risk factors for spinal surgical-site infections in a community hospital: a case-control study. Infect Control Hospital Epidemiol 2003;24:31-6.

12. Mu Y, Edwards JR, Horan TC, Berrios-Torres SI, Fridkin SK. Improving risk-adjusted measures of surgical site infection for the National Healthcare Safety Network. Infect Control Hosp Epidemiol 2011;32:970-86.

13. Anderson D, Podgorny K, Berríos-Torres S, Bratzler DW, Dellinger EP, Greene L, et al. Strategies to prevent surgical site infections in acute care hospitals: 2014 update. Infect Control Hosp Epidemiol 2014;35:605-27.

14. O'Brien G, Buchkley K, Vanwalleghem G, Vanrenterghem D, Dharma H, Winter RL, et al. A multi-centre, prospective, clinical in-market evaluation to assess the performance of Opsite ${ }^{\mathrm{TM}}$ Post-Op Visible dressings. Int Wound J 2010;7, 239-37.

15. Patel N, Bagan B, Vadera S, Maltenfort MG, Deutsch H, Vaccaro AR, et al. Obesity and spine surgery: relation to perioperative complications. J Neurosur Spine 2007;6:291-7.

16. Cao J, Kong L, Meng F, Zhang Y, Shen Y. Impact of obesity on lumbar spinal surgery outcomes. J Clin Neurosci 2016;28:1-6.

17. De la Garza-Ramos R, Bydon M, Abt NB, Sciubba DM, Wolinsky JP, Bydon A, et al The impact of obesity on short-and long-term outcomes after lumbar fusion. Spine (Phila Pa 1976) 2015;40:56-61.

18. Cizik AM, Lee MJ, Martin BI, Bransford RJ, Bellabarba C, Chapman JR, et al. Using the spine surgical invasiveness index to identify risk of surgical site infection. A multivariate analysis. J Bone Joint Surg Am 2012;94:335-42.

19. Abdallah DY, Jadaam MM, McCabe JP. Body mass index and risk of surgical site infection following spine surgery: a meta-analysis. Eur Spine J 2013;22:2800-9.

20. Fei Q, Li J, Lin J, Li D, Wang B, Meng H, et al. Risk factors for surgical site infection after spinal surgery: a meta-analysis. World Neurosurg 2016;95:507-15.

21. Elgafy H, Hamilto R, Peters N, Paull D, Hassan A. Critical care of obese patients during and after spine surgery. World J Crit Care Med 2016;5:83-8.

22. Adogwa O, Elsmadice AA, Fialkoff J, Cheng J, Karikari IO, Bagley C. Early ambulation decreases length of hospital stay, perioperative complications and improves functional outcomes in elderly patients undergoing surgery for correction of adult degenerative scoliosis. Spine 2017;42:1420-5.

23. Ferri T, Valour F, Lustig S, Laurent F, Perrin G, Chidiac C, et al. The challenge of infection prevention in spine surgery: an update. Eur J Ortho Surg Traumatol 2013;23(Suppl 1):S15-9.

24. Agarwal N, Agarwal P, Querry A, Mazurkiewicz A, Tempel ZJ, Friedlander RM, et al Implementation of an infection prevention bundle and increased physician awareness improves surgical outcomes and reduces costs associated with spine surgery. J Neurosurg Spine 2018;29:108-14.

25. Gould JM, Hennessey P, Kiernan A, Safier S, Herman M. A novel prevention bundle to reduce surgical site infections in pediatric spinal fusion patients. Infect Control Hosp Epidemiol 2016;37:527-34. 